\title{
Du dessin à la restitution virtuelle, l'utilisation des nouvelles technologies dans l'étude de la sculpture du haut Moyen Âge
}

From drawing to virtual restitution: the use of new technologies in the study of early medieval sculpture

\section{Jessy Crochat et Anne Flammin}

\section{(2) OpenEdition}

\section{Journals}

Édition électronique

URL : https://journals.openedition.org/cel/20064

DOI : $10.4000 /$ cel.20064

ISSN : 2262-208X

\section{Éditeur}

École du Louvre

\section{Référence électronique}

Jessy Crochat et Anne Flammin, «Du dessin à la restitution virtuelle, l'utilisation des nouvelles technologies dans l'étude de la sculpture du haut Moyen Âge », Les Cahiers de l'École du Louvre [En ligne], 17 | 2021, mis en ligne le 22 novembre 2021, consulté le 13 décembre 2021. URL : http:// journals.openedition.org/cel/20064; DOI : https://doi.org/10.4000/cel.20064

Ce document a été généré automatiquement le 13 décembre 2021.

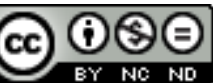

Les Cahiers de l'École du Louvre sont mis à disposition selon les termes de la licence Creative Commons Attribution - Pas d'Utilisation Commerciale - Pas de Modification 4.0 International. 


\section{Du dessin à la restitution virtuelle, l'utilisation des nouvelles technologies dans l'étude de la sculpture du haut Moyen Âge}

From drawing to virtual restitution: the use of new technologies in the study of early medieval sculpture

Jessy Crochat et Anne Flammin

1 L'objectif de cet article est de proposer un état des lieux des nouvelles technologies disponibles à ce jour pour l'étude de la sculpture du haut Moyen Âge. Il s'appuiera en partie sur les expériences menées dans le cadre de nos propres travaux sur la sculpture. Face à l'évolution très rapide des nouvelles technologies, il faut souligner que, dans un futur proche, les outils présentés ici deviendront rapidement obsolètes.

\section{Le dessin ou le relevé : intérêt et limites}

2 Les archéologues ou les historiens de l'art, confrontés à l'étude d'une pièce sculptée, sont amenés, quand ils en ont la possibilité, à réaliser un relevé manuel de l'objet (fig. 1). Munis d'un crayon de bois, d'une planche à dessin avec un calque polyester millimétré, ils procèdent au dessin en prenant des mesures sur l'objet, car le relevé manuel permet une observation complète de l'ensemble des données indispensables à l'examen. Certains détails, comme les restes de pigment ou les traces laissées par un outil de taille, ne sont en effet observables qu'à l'œil nu. Le relevé va alors traduire fidèlement l'observation de l'objet. Il tient compte de ses dimensions réelles et permet d'enregistrer ses caractéristiques (nature du matériau, traces d'outils...) et ses irrégularités (cassures, remploi...). Il devient un document de travail avec des codes de représentation graphique normalisés et des commentaires associés. Il est le support le mieux adapté à l'exercice de la réflexion que doit mener l'archéologue ou l'historien de 
l'art et, associé à un cliché photographique, il formera l'illustration nécessaire à sa présentation.

Fig. 1
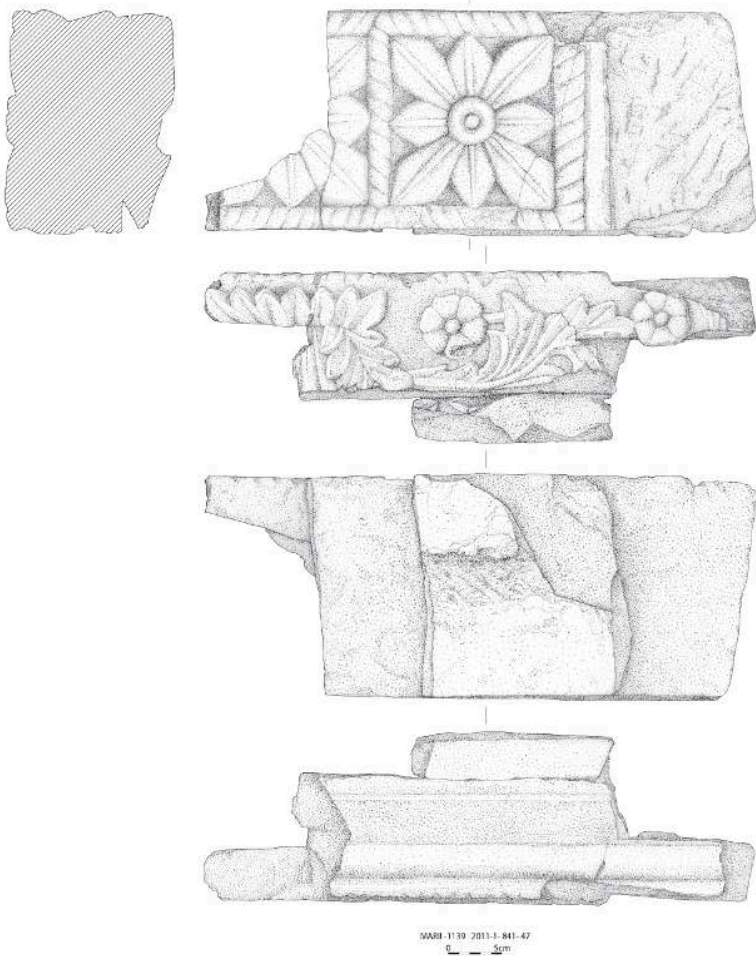

Mariana (Corse), basilique paléochrétienne, relevé d'une architrave d'un ciborium du haut Moyen Âge.

(c) réalisation Marie-Noëlle Baudrand

\section{Du relevé manuel aux relevés lasergrammétrique et photogrammétrique}

Si le relevé manuel reste l'outil le plus pertinent, les chercheurs ont recours, depuis peu, à de nouvelles technologies comme la photogrammétrie ou la lasergrammétrie qui sont deux techniques de numérisation 3D. Elles produisent des données 3D brutes de nature identique : des nuages de points, colorés ou non, dont la position est définie dans l'espace par des coordonnées $\mathrm{x}$, y et $\mathrm{z}$ (fig. 2). Sans prétendre ici expliquer en détail le fonctionnement de ces technologies, rappelons seulement que la lasergrammétrie fait appel à des capteurs numériques motorisés, ou scanners, qui vont permettre de relever des points en coordonnées en enregistrant certaines informations radiométriques. Pour saisir et calculer ces points en $\mathrm{x}$, y et $\mathrm{z}$, il est primordial d'obtenir des mesures de distances et des valeurs angulaires. Il existe plusieurs types de laser scanner dont le design et les performances sont adaptés à la taille du sujet à numériser et à la résolution spatiale souhaitée (sites, objets.... ${ }^{1}$. Dans le cadre de l'étude d'une sculpture isolée (sarcophage...) ou déposée (élément d'une barrière liturgique...), l'emploi d'un scanner à bras pour acquérir le nuage de points apparaît la solution la plus adaptée. L'une des limites de la lasergrammétrie est le coût du matériel, jusqu'à plusieurs dizaines de milliers d'euros pour l'achat d'un laser scanner. Cependant il est 
des domaines d'application où il demeure difficilement remplaçable comme dans le cas d'acquisition des données numériques dans des espaces très sombres (espace semienterré ou souterrain...).

Fig. 2

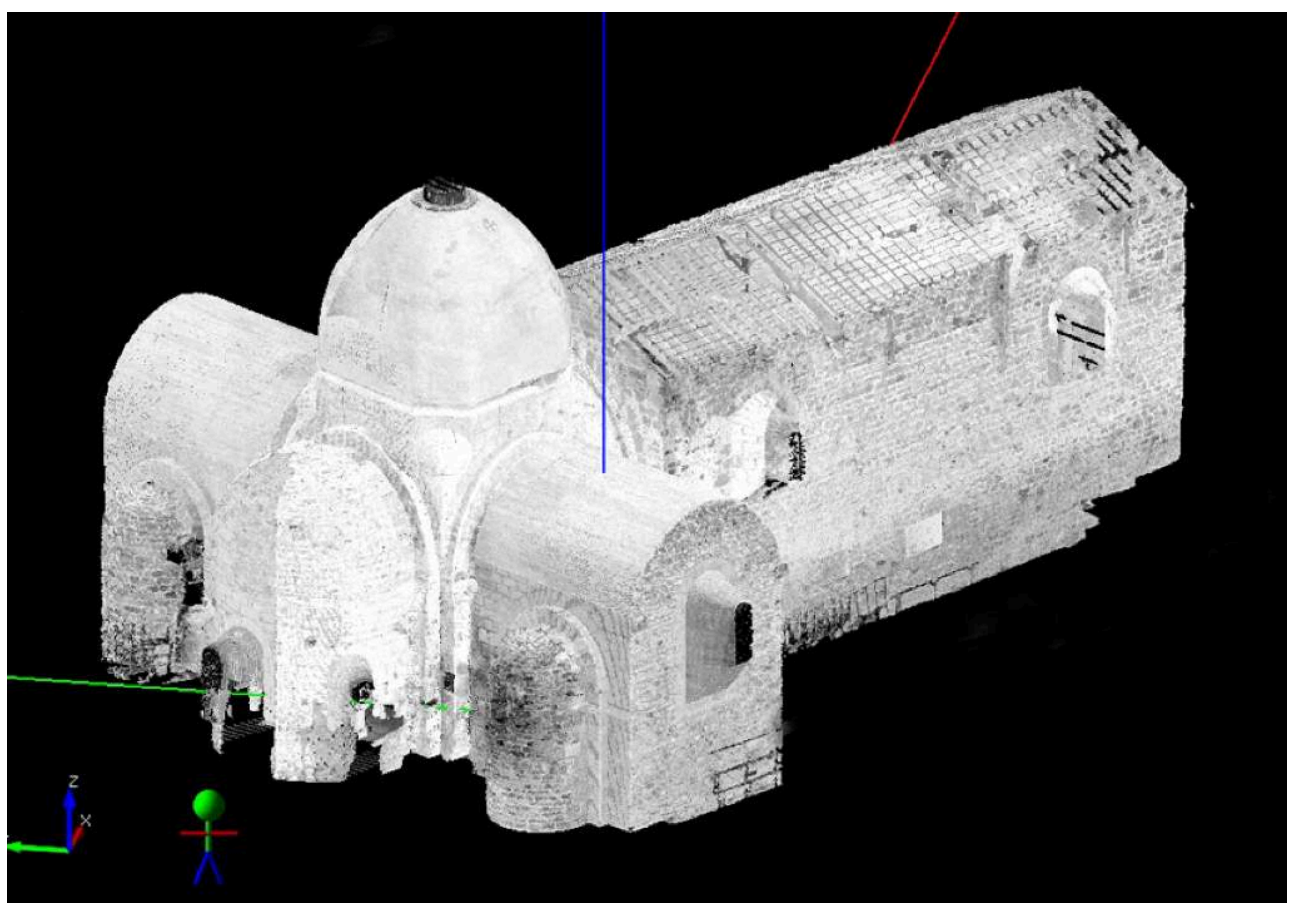

Veyrines (Ardèche), église Sainte-Marie, acquisition d'un nuage de point avec un laser scanner.

(c) réalisation Anne Flammin

4 Grâce au développement, ces dix dernières années, de la photogrammétrie, il est désormais possible d'obtenir rapidement, avec un matériel léger et peu coûteux, un appareil photographique et un logiciel de traitement, un résultat scientifiquement exploitable. On peut même parler d'une véritable révolution dans l'utilisation de la 3D depuis que les archéologues se sont emparés de cet outil. La photogrammétrie est fondée sur un principe inspiré de la vision humaine; cette technique consiste en l'acquisition numérique de plusieurs photographies du même objet prises à partir de points de vue différents ${ }^{2}$. Plusieurs dizaines de clichés, pris sous des angles différents afin de couvrir complétement l'objet, sont utiles pour rendre compte de la complexité d'une sculpture. Le logiciel recherche les points identiques entre les images ${ }^{3}$. Après calcul, il situe les images les unes par rapport aux autres dans un système géographique local et circonscrit tous les points communs à plusieurs d'entre elles dans l'espace. Il compile ainsi ces images pour produire un nuage de points en trois dimensions qui sera maillé, c'est-à-dire que les points sont reliés pour former une surface et procurer ainsi une empreinte virtuelle, une copie conforme de l'objet. Enfin, le résultat est texturé au moyen des photographies, délivrant un modèle 3D en couleur dont l'orientation dans l'espace et l'échelle sont connus, comme par exemple pour le socle des larrons de l'hypogée de Mellebaude à Poitiers (fig. 3$)^{4}$. La qualité du résultat final dépend de la qualité des photos, du nombre et de la pertinence des angles de vue utilisés pour le calcul. Les nuages de points générés constituent de véritables empreintes numériques de l'objet. Il est alors possible d'extraire, à partir de ce nuage, des coupes, des profils et 
des orthophotographies ou ortho-images. L'ortho-image, qui intègre réalité photographique et réalité métrique, est riche en informations.

Fig. 3
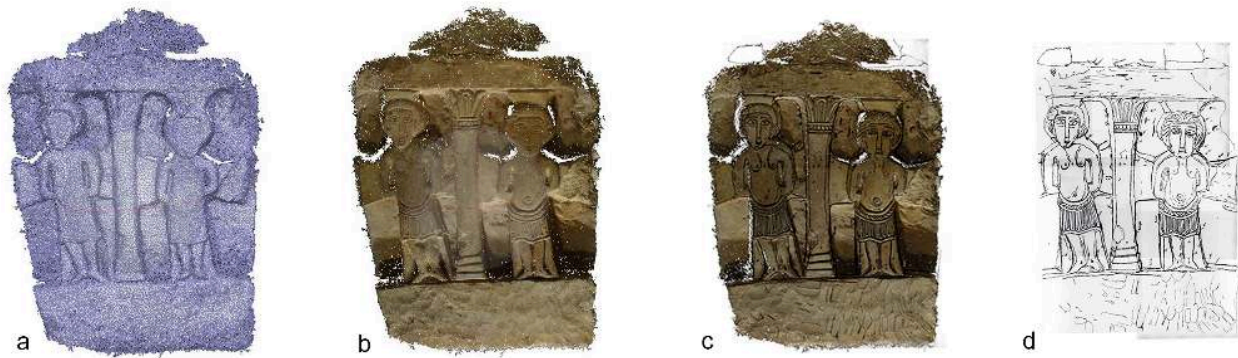

Poitiers (Vienne), hypogée, socle avec les larrons. Poitiers, musée Sainte-Croix, principales étapes du traitement photogrammétrique et dessin : a. Maillage 3D filaire ; b. Modèle 3D texturé ;

c. Orthophotographie ; d. Relevé à partir de l'orthophotographie.

(c) réalisation Anne Flammin

\section{Les avantages des acquisitions lasergrammétriques et photogrammétriques}

Le recours à la lasergrammétrie ou à la photogrammétrie dans le cadre de l'étude de la sculpture offre une rapidité d'acquisition et une grande précision des données. Ces deux technologies offrent aussi la possibilité d'un accès ultérieur à la copie 3D de la pièce qui peut être manipulée virtuellement comme dans l'exemple du relevé photogrammétrique d'un fragment d'une table d'autel découvert à Nice (fig. 4). Ce fragment a été retrouvé en position de remploi dans un caveau du cimetière attenant à l'ancienne cathédrale de Nice, située sur la colline du Château ${ }^{5}$. L'accès à la copie en 3D de l'objet permet d'en faire une analyse ou d'en extraire des vues ou des profils sans manipulation de l'original et à distance. Sur les vues ou sur l'orthophotographie extraites, comme sur le socle des larrons de l'hypogée de Mellebaude (fig. 3, c.), il est possible, par exemple, de zoomer et de visualiser les traces laissées par les outils de taille ou encore prendre des dimensions. L'opportunité de produire une archive à un « instant $\mathrm{T}$ » de la sculpture peut également être avantageux. Les relevés 3D peuvent aussi aider au diagnostic de l'état des œuvres : dans le cadre de la conservation et de la gestion des objets muséaux par exemple, il sert à localiser et à mesurer les altérations de tous ordres et de situer toute intervention, analyse ou prélèvement. Les travaux menés par des restaurateurs en archéologie ont démontré l'intérêt de l'utilisation de la photogrammétrie dans le cadre d'œuvres sculptées dont l'état de conservation médiocre (en plusieurs parties) nécessite de limiter les manipulations ${ }^{6}$. La visualisation et la manipulation virtuelle des différentes pièces leur a permis par exemple d'anticiper les dispositifs nécessaires à la restauration et de réaliser un soclage aux bonnes dimensions pour la présentation muséale. 
Fig. 4

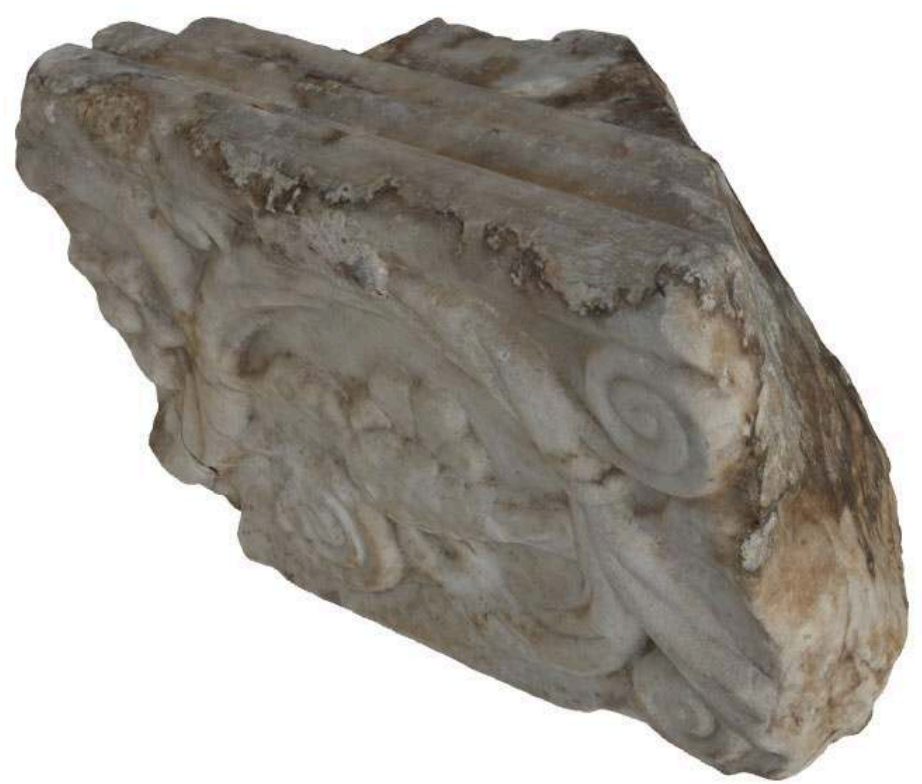

Nice (Alpes-Maritimes), table d'autel fragmentaire, relevé photogrammétrique (c) réalisation Jessy Crochat.

Ce type d'acquisitions oblige aussi à "penser en 3D » et permet de tester des hypothèses de restitution qui peuvent être communiquées facilement à d'autres chercheurs. Dans le cadre de l'étude du mobilier liturgique découvert à Vienne (Isère), l'un des auteurs du présent article a, par exemple, proposé une restitution d'une plaque de chancel et de ses différentes attaches pour réfléchir à son intégration et à son positionnement dans la barrière entière (fig. 5) ${ }^{7}$.

Fig. 5

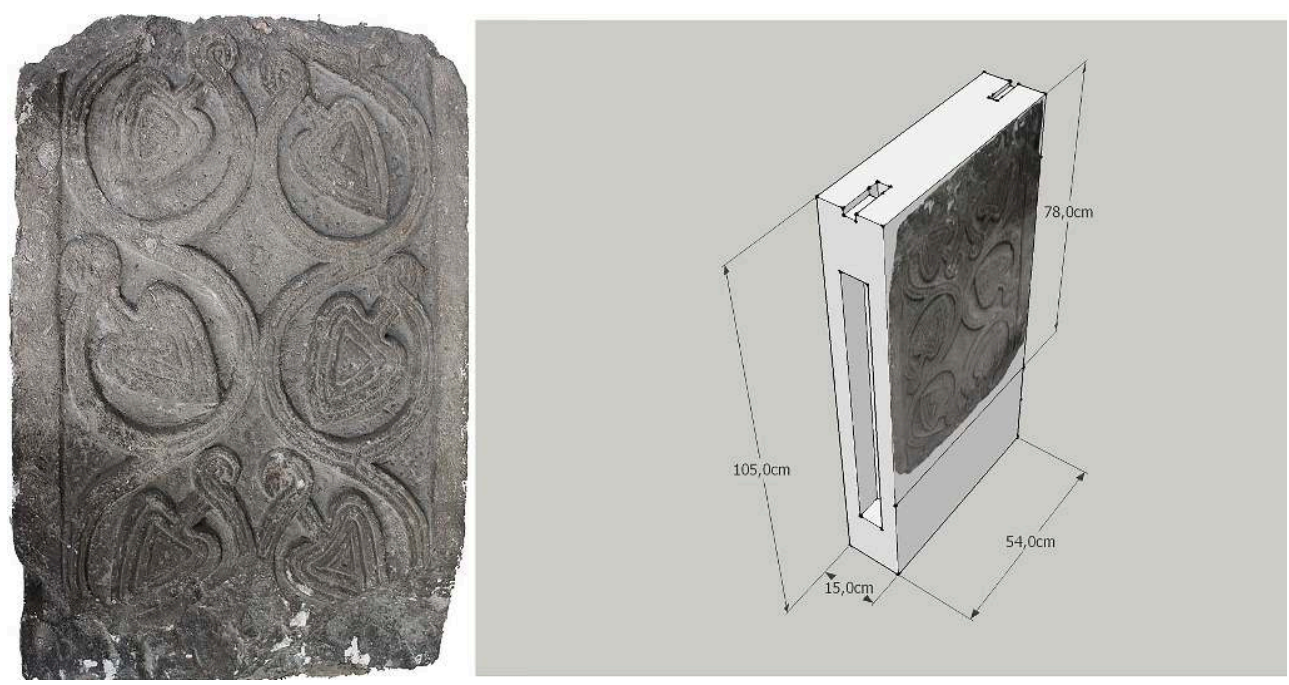

Vienne (Isère), plaque de chancel, restitution avec le logiciel SketchUp et insertion d'une orthophotographie.

(c) réalisation Jessy Crochat. 
7 La valeur heuristique des restitutions 3D a donc fait son chemin dans la communauté scientifique. Certains néanmoins se méfient encore de «l'effet de réel " qu'elles produisent et de la difficulté qu'il y a, sur une image, à départager les différents niveaux de certitudes et d'hypothèses de restitution.

Enfin, si ces outils s'avèrent pertinents pour le travail de réflexion des archéologues sur des vestiges lacunaires, ils le sont aussi, évidemment, pour la médiation à destination $\mathrm{du}$ grand public. Un nouvel exemple est fourni par le travail de restitution d'un sarcophage découvert lors des fouilles de la basilique funéraire Saint-Martin de Luxeuil (Haute-Saône) que Sébastien Bully, chercheur au CNRS, a dirigées de 2005 à 2015 (fig. 6) ${ }^{8}$. Les différents fragments ont d'abord été traités en photogrammétrie par Jessy Crochat pour servir à leur modélisation, réalisée par Jean-Clément Turblin de la société Motion Agency, et à leur positionnement dans le couvercle restitué. À partir de ce remontage, il a été possible de proposer une restitution du décor entier, sur la base de comparaison avec des motifs identiques connus sur d'autres sarcophages mérovingiens, comme ceux des exemplaires découverts à Chalon-sur-Saône (Saône-et-Loire), à Cluny (Saône-et-Loire) et à Villars-Saint-Marcellin (Haute-Marne). Ce travail, une fois terminé et la première version amendée, sera intégré à la présentation du site dans le musée de Luxeuil. Il a nécessité de constants échanges entre les archéologues et la société chargée de la modélisation pour tester les différentes hypothèses. La restitution 3D fait donc maintenant partie de la chaîne opératoire de l'archéologie et offre l'opportunité, dans cet exemple, de proposer au public les deux hypothèses envisagées pour l'emplacement de ce sarcophage dans la crypte de Saint-Valbert : sur une banquette, à l'est, ou au centre. La restitution 3D est donc tout autant un support de réflexion et d'expérimentation que de communication des travaux.

Fig. 6

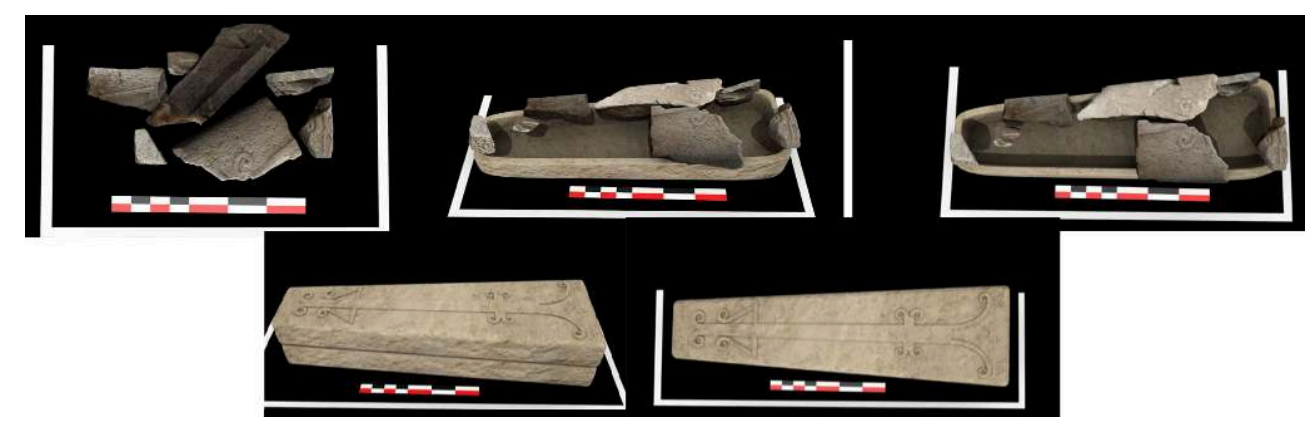

Luxeuil (Haute-Saône), ancienne église Saint-Martin, sarcophage découvert en fouille par Sébastien Bully (CNRS-UMR ARTEHIS) - 2005-2015. Premières restitution 3D par Jean-Clément Turblin de la société Motion Agency.

(c) Jean-Clément Turblin / Motion Agency

9 À une plus grande échelle et dans le domaine de la sculpture romane cette fois, on pourra également évoquer le travail de reconstitution de la façade de la tribune de l'abbaye Saint-Michel de Cuxa (Pyrénées-Orientales) ${ }^{9}$. Sur la base de la modélisation numérique réalisée par Anna Thirion dans le cadre de sa thèse, l'anastylose virtuelle de ce monument disparu a été possible à partir du remontage des éléments qui en proviennent, dispersés en France et à l'étranger. L'anastylose virtuelle présente l'intérêt de les réunir dans un même espace, de matérialiser des hypothèses d'assemblage et de les partager avec tous les publics. 
10 La 3D est également un outil pédagogique très intéressant. Le relevé 3D peut aussi servir de base à la fabrication de fac-similés virtuels ou physiques, comme support de produits culturels, éducatifs ou touristiques à destination de tous les publics.

\section{L'utilisation de Reflectance Transformation Imaging ou RTI pour l'étude de la sculpture}

11 Enfin, parmi les nouvelles technologies applicables à l'étude de la sculpture, il faut mentionner la Reflectance Transformation Imaging ou RTI (imagerie par transformation de la réflectance), une méthode photographique développée au début des années 2000 par le Cultural Heritage Imaging ${ }^{10}$. Elle désigne un ensemble de techniques basées sur la photographie qui permet d'acquérir et de visualiser des informations sur la façon dont un objet ou une scène interagit avec la lumière. Le principe est d'acquérir une série de photographies d'un objet depuis un point de vue fixe, mais en faisant varier la direction de l'éclairage d'une image à l'autre. Le modèle ainsi obtenu pourra être utilisé pour générer de nouvelles images avec des directions d'éclairage différentes de celles utilisées lors de l'acquisition, et ce de façon interactive. On peut également en extraire des informations sur la forme de l'objet, ou encore modifier ses propriétés pour faire ressortir certains détails.

Ainsi les détails invisibles à l'œil nu apparaissent et participent à l'étude tracéologique de la sculpture. Cette technique s'est révélée extrêmement utile dans le cadre de l'étude d'une tablette gravée médiévale découverte en 2017 dans une sépulture du site d'Ossor en Croatie, dont les travaux archéologiques sont dirigés par Sébastien Bully, Morana Čaušević-Bully, Iva Maric et Miljenko Jurkovic. Les différentes images réalisées par Fabrice Monna et Tanguy Rolland de l'UMR Artehis permettent de lire parfaitement le décor et de faire apparaître des détails qui échappent parfois à l'œil nu (fig. 7) : traces laissées par les outils de taille de pierre, éventuels repentirs... Elle est évidemment particulièrement intéressante en épigraphie, pour améliorer la lecture des inscriptions.

Fig. 7
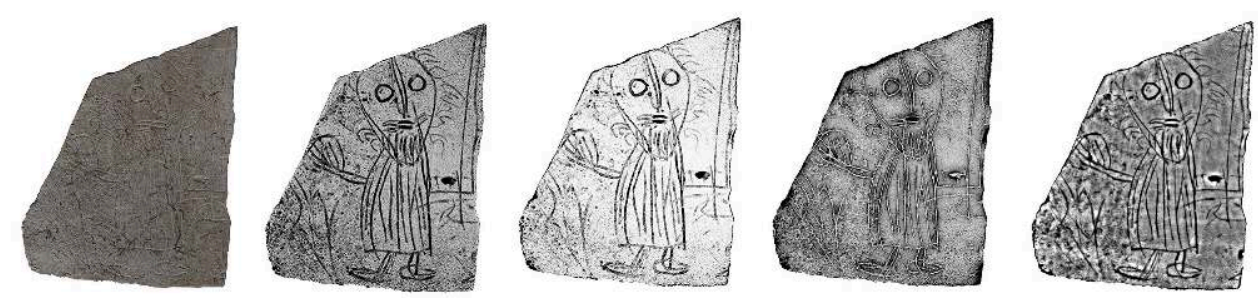

Osor (Croatie), monastère Saint-Pierre, tablette médiévale gravée découverte dans une tombe (Xle-XIle s. ?). Fouilles dirigées par Sébastien Bully, Morana Čaušević-Bully, Iva Maric et Miljenko Jurkovic. Travaux RTI : Fabrice Monna, Tanguy Rolland (Umr Artehis-Université de Bourgogne).

(c) Travaux RTI : Fabrice Monna, Tanguy Rolland (Umr Artehis-Université de Bourgogne).

En conclusion, et sans nier l'apport indéniable de la lasergrammétrie et de la photogrammétrie à l'étude de la sculpture, il est nécessaire de souligner leurs limites et de rappeler que l'innovation et le haut degré de technicité de ces outils ne doivent pas faire oublier que les résultats obtenus peuvent être insuffisants et nécessitent très souvent le recours au relevé traditionnel fondé sur une observation directe de la 
sculpture. Le relevé manuel ne doit pas être abandonné au profit unique de ces nouveaux outils et un usage conjoint est recommandé. Il faut surtout mettre en garde les utilisateurs sur le fait que la technique ne peut pas se substituer à la réflexion et qu'un très beau modèle 3D ne remplace jamais l'analyse de l'objet. De plus, la modélisation d'objets en 3D est chronophage et peut rapidement empiéter sur le temps dédié à l'analyse. Or, ce temps est incompressible pour des études qualitatives. L'utilisation de ces nouvelles technologies ne doit pas faire perdre de vue l'objet d'étude. Dans de nombreux cas, un relevé manuel suffit. Plus encore, il offre une réelle connexion entre l'objet et le chercheur qui devra se concentrer sur l'élément analysé pour en reporter les moindres détails sur le papier.

\section{NOTES}

1. On trouve ainsi des scanners laser dits à longue portée, qui sont le plus souvent à technologie temps de vol et ont une résolution spatiale adaptée aux sujets de grandes dimensions (quelques centimètres à quelques millimètres); et des scanners à courte portée, qui exigent d'être près du sujet et dont la résolution peut descendre en dessous du millimètre, jusqu'à quelques dizaines de microns.

2. Sur la photogrammétrie : Raphaële Héno, Laure Chandelier, Numérisation 3D de bâtiments : Cas des édifices remarquables, Paris, Iste Éditions, 2014.

3. Parmi les outils les plus utilisés on pourra citer le logiciel payant Metashape, développé par Agisoft, et Micmac un logiciel libre proposé par l'IGN.

4. L'acquisition par photogrammétrie du socle dit des larrons, conservé au musée Sainte-Croix de Poitiers, a été réalisée dans le cadre de l'étude archéologique de l'hypogée entrepris par une équipe pluridisciplinaire dirigée par Cécile Treffort et Bénédicte Bertholon.

5. Sur ce fragment, conservé dans les réserves du service archéologique de Nice, on pourra consulter le rapport de fouille annuel du PCR «La colline du Château à Nice » de l'année 2016, sous la direction d'Aude Civetta. Voir également Fabien Blanc-Garidel, L'archéologie entre Monts et Rivages, t. 2 Moyen Âge, Époque moderne, Époque contemporaine, Ville de Nice, 2020, pp. 21 et 111.

6. Sur l'expérience menée par Hélène Collomb et Anaïs Lechat dans le cadre de la restauration d'un atlante daté du $\mathrm{II}^{\mathrm{e}}$ siècle conservé à Bruxelles, on consultera : Hélène Collomb, Anaïs Lechat, «Mise en valeur des sculptures fragmentaires en matériaux pierreux, en contexte muséal: la photogrammétrie comme outil d'aide à la décision", Actes des Journées des restaurateurs en archéologie organisées par le laboratoire Arc'Antique, Nantes, 15 et 16 octobre 2015, Cahier technique, $\mathrm{n}^{\circ} 23$, 2018, pp. 87-95.

7. La restitution a été réalisée avec le logiciel gratuit Sketchup et l'application d'une orthophotographie obtenue avec le logiciel Metashape. Sur ce travail : Jessy Crochat, Le mobilier liturgique dans les diocèses médiévaux de Grenoble, Lyon et Vienne IV ${ }^{e}-X I^{e}$ siècles, Mémoire de master sous la direction d'Anne Baud, Université Lumière Lyon 2, 2015.

8. Sébastien Bully, Aurélia Bully (coll.), «La crypta mérovingienne de l'église Saint-Martin de Luxeuil et la tombe de l'abbé Walbert ", Saint Walbert, le rayonnement du monachisme luxovien dans le royaume franc au VII siècle, Les Cahiers Colombaniens, à paraître (2021).

9. Ce travail s'est appuyé sur un inventaire et une étude des sculptures. Sur un modèle filaire, qui est une représentation de la structure en 3D uniquement faite de lignes et de courbes et qui 
forme donc un squelette de l'ensemble, ont été disposés les éléments numérisés. Plus de 170 sculptures ont été numérisées par photogrammétrie. Le tracé se base bien évidemment sur l'étude des vestiges archéologiques (traces de l'emplacement originel) et sur celle des sculptures conservées : http://cuxa.org/

10. Cultural Heritage Imaging est une société à but non lucratif qui a pour objectif de développer l'utilisation des nouvelles technologies en lien avec l'image. http://culturalheritageimaging.org

\section{RÉSUMÉS}

Si le relevé manuel reste l'outil le plus souvent pertinent dans le cadre de l'étude de la sculpture, les archéologues ont recours, depuis peu, à de nouvelles technologies comme la photogrammétrie, la lasergrammétrie ou la RTI (Reflectance Transformation Imaging). Les nuages de points générés constituent de véritables empreintes numériques dont on peut extraire des coupes, des profils ou des orthophotographies. Il est possible de restituer des parties disparues des sculptures et de réaliser des assemblages, souvent impossibles à mettre en œuvre autrement. Le modèle virtuel permet également de tester les hypothèses. Nous étudierons donc, à travers différents exemples issus, pour la plupart, de nos propres travaux, les avantages et les inconvénients de ces nouveaux relevés numériques.

Although manual surveying is still the most relevant tool for the study of sculpture, archaeologists have recently started using new technologies such as photogrammetry, lasergrammetry and reflectance transformation imaging (RTI). The scatter charts generated constitute digital prints from which cross-sections, profiles and orthophotographs may be extracted. It is possible to restore missing parts of the sculptures and to create assemblages that are often impossible to implement otherwise. The virtual model also makes it possible to test hypotheses. We will thus study the advantages and disadvantages of these new digital surveys using various examples, most of which come from our own work.

\section{INDEX}

Mots-clés : sculpture, modèle 3D, laser scanner, lasergrammétrie, photogrammétrie, RTI (Reflectance Transformation Imaging)

Keywords : sculpture, 3D model, laser scanner, lasergrammetry, photogrammetry, reflectance transformation imaging (RTI)

\section{AUTEURS}

\section{JESSY CROCHAT}

Jessy Crochat, chercheur associé à l'UMR ARTEHIS, a soutenu un mémoire de master, Le mobilier liturgique dans les diocèses médiévaux de Grenoble, Lyon et Vienne du IVe au XIe siècle, sous la direction d'Anne Baud et la tutelle d'Anne Flammin à l'université Lumière Lyon 2 en 2015. Il est actuellement archéologue du bâti au sein de l'entreprise Archeodunum SAS. Il étudie le mobilier 
lapidaire de plusieurs sites archéologiques en Croatie. Il fait également partie du programme ANR Monacorale sur les complexes ecclésiaux et monastiques côtiers et insulaires de l'Adriatique du IV ${ }^{\mathrm{e}}$ au XI ${ }^{\mathrm{e}}$ siècle, porté par Sébastien Bully, Morana Čaušević-Bully, Stéphane Gioanni et Pascale Chevalier.

Jessy Crochat, a researcher for UMR ARTEHIS, defended a master's dissertation, "Le mobilier liturgique dans les diocèses médiévaux de Grenoble, Lyon et Vienne du $\mathrm{IV}^{\mathrm{e}}$ au XI $\mathrm{XI}^{\mathrm{e}}$ siècle", supervised by Anne Baud and Anne Flammin, at the Université Lumière Lyon 2 in 2015. He currently employed as an archaeologist for the company Archeodunum SAS. He is studying the lapidary furniture of several archaeological sites in Croatia. He is also part of the ANR Monacorale programme on the coastal and insular ecclesiastical and monastic complexes of the Adriatic from the 4th to the 11th century, headed by Sébastien Bully, Morana Čaušević-Bully, Stéphane Gioanni and Pascale Chevalier.

\section{ANNE FLAMMIN}

Anne Flammin, membre du projet SATHMA, a soutenu une thèse de doctorat, La sculpture du haut Moyen Âge entre Loire et Gironde sous la direction du Professeur Piotr Skubiszewski au Centre d'Études Supérieures de Civilisation Médiévale de Poitiers en 1999. Elle est aujourd'hui Ingénieure de recherche en archéologie au CNRS dans l'UMR 5138 « Archéologie et archéométrie » à Lyon. Elle co-dirige plusieurs chantiers d'archéologie médiévale en France (abbaye de Cluny, de Saint-André-le-Haut à Vienne...) et participe à des missions de fouilles à l'étranger, notamment au Proche-Orient.

Anne Flammin, member of the SATHMA project, supported a doctoral thesis, "La sculpture du haut Moyen âge entre Loire et Gironde", supervised by Professor Piotr Skubiszewski, at the Centre d'Études Supérieures de Civilisation Médiévale de Poitiers in 1999. She is currently an archaeological researcher for the CNRS (UMR 5138, Archéologie et archéométrie, Lyon. She helps direct several medieval archaeological projects in France (Abbaye de Cluny, Abbaye de SaintAndré-le-Haut à Vienne, etc.) and takes part in excavations abroad, particularly in the Middle East. 